\title{
CARACTERÍSTICAS DEL DIAGNÓSTICO Y TRATAMIENTO DE LA TUBERCULOSIS PULMONAR EN PACIENTES CON Y SIN DIABETES MELLITUS TIPO 2
}

\author{
Omar Carrión-Torres ${ }^{1, a}$, Patrick Cazorla-Saravia ${ }^{1, a}$, José William Torres Sales ${ }^{1, b}$, Nilton Yhuri Carreazo ${ }^{1, c}$, \\ Frank Enrique De La Cruz Armijo ${ }^{1, a}$
}

\begin{abstract}
RESUMEN
Objetivos. Conocer si existen diferencias en las características demográficas, clínicas y radiológicas entre los pacientes con tuberculosis pulmonar (TB) y pacientes con TB y diabetes mellitus tipo 2 (TB+DM2). Materiales y métodos. Estudio observacional de cohorte retrospectiva. Se compararon las características clínicas de los pacientes según el sexo, la edad, el tiempo de negativización del esputo, la presencia de cavitación así como la tasa de curación, duración del tratamiento y la proporción del cambio en el esquema del tratamiento antituberculoso en pacientes con y sin DM2 atendidos por el Programa de Control de Tuberculosis entre 2010 y 2012 en la Red Asistencial Rebagliati de Lima, Perú. Resultados. Se incluyeron 31 pacientes con TB+DM2 y 144 pacientes con TB. Se encontraron diferencias $(p<0,05)$ en el método de diagnóstico, el promedio de síntomas y en el patrón de resistencia de TB entre los pacientes con y sin DM2. La presencia de cavitación fue más frecuente en los pacientes con TB+DM2; ser paciente de TB+DM2 retraso la negativización del BK de esputo (RRa 4,16; IC 95 \%: 1,1-1,6) en el análisis de regresión de Cox ajustado. Conclusiones. Existen diferencias en las características demográficas, clínicas y radiológicas en los pacientes con tuberculosis con y sin DM2. El periodo de negativización del esputo es mayor en los pacientes con DM2.
\end{abstract}

Palabras clave: Tuberculosis; Diabetes mellitus tipo 2; Estudios longitudinales; Comorbilidad; (fuente: DECS BIREME).

\section{CHARACTERISTICS OF THE DIAGNOSIS AND TREATMENT OF PULMONARY TUBERCULOSIS IN PATIENTS WITH AND WITHOUT DIABETES MELLITUS TYPE 2}

\begin{abstract}
Objectives. To determine whether there are demographic, clinical and radiological differences among patients with pulmonary tuberculosis (TB) and patients with TB and type 2 diabetes mellitus (DM2 + TB). Materials and methods. Observational retrospective cohort study. We compared the clinical characteristics of patients according to sex, age, time to sputum conversion to negative, presence of cavitation and the cure rate, duration of treatment and the proportion of change of treatment regimen, in patients with and without DM2 served by the Tuberculosis Control Program from 2010 to 2012 in the Rebagliati Healthcare Network of Lima, Peru. Results. 31 patients with TB+DM2 and 144 patients with TB were included. Differences $(p<0.05)$ in the diagnostic method, the average of symptoms and the resistance pattern of TB among patients with and without DM2 were found. The presence of cavitation was more frequent in patients with TB + DM2. Having TB + DM2 delayed the time to sputum smear conversion to negative (RRa 4.16, $95 \% \mathrm{Cl}: 1.1-1.6)$ in the adjusted Cox regression analysis. Conclusions. There are differences in demographic, clinical and radiological characteristics in TB patients with and without DM2. The time to sputum conversion to negative is greater in patients with DM2.
\end{abstract}

Key words: Tuberculosis; Diabetes Mellitus, Type 2; Longitudinal studies; Comorbidity (source: MeSH NLM).

\footnotetext{
Escuela de Medicina Humana, Universidad Peruana de Ciencias Aplicadas. Lima, Perú.

Médico cirujano; ${ }^{\mathrm{b}}$ médico neumólogo; ${ }^{\mathrm{c}}$ médico pediatra

Recibido: 23-04-15 Aprobado: 19-08-15
}

Citar como: Carrión-Torres O, Cazorla-Saravia P, Torres Sales JW, Carreazo Pariasca NY, De La Cruz Armijo FE. Características del diagnóstico y tratamiento de la tuberculosis pulmonar en pacientes con y sin diabetes mellitus tipo 2. Rev Peru Med Exp Salud Publica. 2015;32(4):680-6. 


\section{INTRODUCCIÓN}

La tuberculosis (TB) es un problema de salud pública en el mundo. Clínicamente, existen tres formas: la TB pulmonar se caracteriza por tos crónica, esputo, pérdida de peso y de apetito, fiebre, sudoración nocturna y hemoptisis; la forma extrapulmonar puede comprometer otros órganos y presentar diferentes expresiones clínicas y la co-infeccion de TB con otros agentes ${ }^{(1)}$. Desde el punto de vista de la sensibilidad a los medicamentos antituberculosos, la TB se clasifica en pansensible, multidrogorresistente y extensamente resistente ${ }^{(2)}$.

En el año 2012, se estimaron en el mundo 8,6 millones de casos nuevos de TB y 1,3 millones murieron a causa de la enfermedad, de ellos, 450 mil por la TB multidrogorresistente (TB MDR) y 320 mil por co-infección con VIH ${ }^{(3)}$. Perú es uno de los países endémicos tanto para la TB como para la forma MDR, la incidencia acumulada en el 2012 fue 93 casos por 100 000 habitantes y la región Lima, ocupa el quinto lugar entre todas las regiones con una incidencia de 148 por 100000 habitantes ${ }^{(4)}$

Existe un incremento de TB con otras enfermedades como el $\mathrm{VIH}$, desnutrición y diabetes mellitus tipo 2

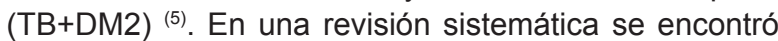
que en los estudios de cohorte, la diabetes incrementa el riesgo de desarrollar la TB activa en más de 3 veces comparado con los que no tienen diabetes, y en los estudios de casos y control los estimadores de riesgo varían desde 1,16 hasta $7,83^{(6)}$. Según los reportes sobre la proporción de los casos de TB+DM2, en los Estados Unidos de América es $11,4 \%$ (7), $13,1 \%$ en Japón ${ }^{(8)}, 29,6 \%$ en México ${ }^{(9)}$ y en el Perú es $6,3 \%{ }^{(10)}$. Si bien la prevalencia de diabetes en Perú es baja, su incremento en los próximos años podría ocasionar el incremento de la comorbilidad TB+DM2 (11,12).

La diabetes es un factor de riesgo importante para enfermar de TB ${ }^{(6,13)}$, debido a la inmunosupresión que ocasiona, algunos estudios plantean que modifica la presentación clínica, bacteriológica y la respuesta al tratamiento, incrementando el riesgo de la resistencia a las drogas y el riesgo de morir ${ }^{(10,14-19)}$.

La asociación TB+DM2 prolonga el tiempo de la baciloscopía (BK) positiva y produce un retraso en la negativización de los cultivos de las micobacterias ${ }^{(9,20,21)}$, lo que implica un mayor tiempo de transmisión en la comunidad. Algunos investigadores estiman que las lesiones radiográficas pulmonares cavitarias entre los pacientes diabéticos son más severas ${ }^{(14)}$. Asimismo, la tasa de fracaso del tratamiento antituberculoso es más alta en los pacientes diabéticos que en los no diabéticos ${ }^{(9,22)}$ y la duración del tratamiento para la TB es más larga en los pacientes con diabetes ${ }^{(17)}$.

Existen pocos estudios que han evaluado las características de los pacientes de tuberculosis con y sin diabetes. Se estima que para el 2030 el $80 \%$ de los pacientes con diabetes vivirán en América Latina, por ello, es importante conocer mejor las características clínicas y epidemiológicas del binomio TB+DM2 para un adecuado tratamiento y control (23).

El objetivo del estudio fue conocer si existen diferencias en cuanto al sexo, la edad y en el tiempo de negativización del esputo, la presencia de cavitación y otras características clínicas y en la tasa de curación, duración del tratamiento y la proporción del cambio en el esquema del tratamiento entre pacientes de tuberculosis pulmonar con y sin diabetes mellitus tipo 2 .

\section{MATERIALES Y MÉTODOS}

\section{DISEÑO DE ESTUDIO}

Estudio de cohorte histórica de pacientes adultos con diagnóstico de TB pulmonar confirmado clínicamente, cultivo o un BK de esputo positivo (24). Se definió como expuesto a un paciente con diagnóstico por baciloscopía y/o cultivo de TB+DM2 y como no expuesto a un paciente solo con diagnóstico por baciloscopía y/o cultivo de TB, ambos, se identificaron en el mismo momento. Los participantes fueron casos nuevos y la fuente de información fueron las historias clínicas.

\section{POBLACIÓN Y TAMAÑO MUESTRAL}

La población del estudio fueron los pacientes atendidos entre enero de 2010 y diciembre de 2012 por los centros médicos de Pablo Bermúdez, Chincha, Hospital Carlos Alcántara, Hospital Angamos y Hospital Uldarico Roca por el Programa de Tuberculosis de la Red Asistencial Rebagliati (Lima, Perú).

El cálculo del tamaño de muestra para un estudio de cohortes se realizó mediante el programa Epilnfo 7.0 a través de la aplicación StatCalc considerando una potencia del $80 \%$, nivel de confianza de $95 \%$ y una proporción de uno (grupo expuesto) a cuatro (grupo no expuesto). Se consideró la proporción de exposición al factor de interés (Resistencia al tratamiento de tuberculosis) entre los expuestos (TB+DM2) 26,1\% y la proporción del factor (Resistencia al tratamiento de tuberculosis) entre los no expuestos (TB) de 5,6\% ${ }^{(17)}$. El tamaño de muestra calculado fue 26 sujetos para el grupo de expuestos, mientras que para el grupo de no expuestos fue 102 sujetos, el total de la muestra a seleccionar en 
ambos grupos fue 128 participantes mediante el método de Fleis. Sin embargo, para tener mayor consistencia se agregó 5 expuestos y 42 no expuestos obteniendo una muestra final de 175 participantes.

\section{DEFINICIÓN DE VARIABLES}

Los esquemas de tratamiento de TB del Ministerio de Salud son: $\mathrm{H}$ (isoniazida), $\mathrm{R}$ (rifampicina), $\mathrm{Z}$ (pirazinamida), $\mathrm{E}$ (etambutol) y $S$ (estreptomicina). El Programa de Control de Tuberculosis en EsSalud se rige por la norma técnica del Ministerio de Salud ${ }^{(24)}$, es decir, los pacientes TB sensible son tratados y controlados en su policlínico de referencia con la estrategia denominada programa de vigilancia directa de tratamiento y los pacientes TB multidrogorresistente son tratados y controlados en el hospital de referencia.

Se define como caso de tuberculosis pulmonar (TB) a una persona con baciloscopía y/o cultivo positivo de BK, con compromiso del parénquima pulmonar evidenciado a la radiografía y signos y síntomas compatibles. Se considera caso de TB sensible a un paciente en el que se demuestra sensibilidad a todos los medicamentos de primera línea por pruebas de sensibilidad convencional y caso de TB multidrogorresistente (TB MDR) al paciente con resistencia simultánea a isoniacida y rifampicina por pruebas convencionales ${ }^{(24)}$.

Asimismo, se define como negativización en esputo a la conversión de la prueba de baciloscopía o cultivo positivo de un paciente antes del inicio del tratamiento a negativomedido a los 4 meses del tratamiento. La presencia de reacción adversa a fármacos antituberculosos (RAFA) se considera cuando ocurre alguna reacción adversa a los fármacos antituberculosos registrado en la historia clinica.

El fracaso al tratamiento es definido como la persistencia de la baciloscopía o cultivo positivo al cuarto mes del tratamiento antituberculoso. De acuerdo al programa de TB, todos los pacientes son monitoreados mediante baciloscopía o cultivo en forma mensual hasta que el paciente se hace negativo o hasta los 12 meses en los positivos. El tiempo de tratamiento antituberculoso es definido como la duración de la terapia antituberculosa. El cambio de tratamiento antituberculoso es cualquier variación en las drogas que no esté contemplada en el esquema inicial (24). Finalmente, la presencia de cavitaciones fue diagnosticada durante el tratamiento.

\section{SELECCIÓN DE LOS PARTICIPANTES Y RECOLECCIÓN DE INFORMACIÓN}

Para identificar a los expuestos (TB+DM2) se elaboró una lista de los casos de tuberculosis primaria que hayan culminado el tratamiento que cumplan con la definición de expuesto a partir de la tarjeta de control de asistencia y administración de medicamentos en el Programa de Control de Tuberculosis (PCT) entre enero de 2010 a diciembre de 2012. Una vez identificado el paciente, se recuperó su historia clínica con el diagnóstico de diabetes mellitus tipo 2 de la oficina de archivos de cualquiera de los centros médicos u hospitales de la Red Asistencial Rebagliati de Lima y c). Finalmente, se seleccionaron por conveniencia las historias clínicas de los casos expuestos que cumplan con la definición.

La selección de los no expuestos fue: a). Se elaboró una lista de los casos de tuberculosis que cumplan con la definición de no expuesto a partir de la lista de pacientes atendidos en el PCT con tratamiento cumplido entre los años 2010 a 2012 de la Red Asistencial Rebagliati de Lima, b). Se realizó un muestreo aleatorio simple para seleccionar los pacientes y c). Las historias clínicas seleccionadas fueron ubicadas y se revisaron para verificar que cumplan con la definición. Los datos de las variables de edad, sexo, procedencia, diagnóstico, síntomas, hallazgos radiológicos, tiempo en meses para la negativización, duración en meses y resultado del tratamiento, patrón de resistencia, presencia de reacciones adversas, paciente diabético, tiempo de enfermedad fueron recogidos mediante una ficha.

\section{CRITERIOS DE SELECCIÓN}

Se consideró como criterios de inclusión del grupo de no expuestos a los pacientes con diagnóstico de TB pulmonar confirmado por cultivo y/o un BK de esputo positivo, que culminaron el tratamiento, además, que no cumpla ningún criterio para el diagnóstico de DM2 de acuerdo a las normas de la Asociación Latinoamericana de Diabetes (ALAD) ${ }^{(25)}$

Se consideró como criterios de inclusión del grupo de expuestos (TB+DM2) a los pacientes con diagnóstico de TB pulmonar confirmado por cultivo y/o un BK de esputo positivo, que culminaron el tratamiento; además con el registro en la historia clínica del diagnóstico de DM2. Se excluyeron a los pacientes con antecedente previo de TB y/o alguna condición inmunosupresora como VIH, cáncer o uso de corticoides a dosis inmunosupresora y a los casos de TB identificados únicamente por criterio clínico. Además, se excluyeron a los pacientes que abandonaron el tratamiento o fueron transferidos sin información a término para evaluar únicamente a los que han concluido el tratamiento brindado.

\section{ANÁLISIS ESTADISTICO}

Los datos fueron ingresados en una base de datos en el programa Microsoft Excel 2010. Los pacientes que abandonaron el tratamiento, los casos diagnosticados 
solo por la clínica o fueron transferidos fueron excluidos del análisis, dado que el interés fue evaluar únicamente a los que han concluido el tratamiento brindado. Para el análisis de variables categóricas se empleó la prueba de chi cuadrado y se consideró como estadísticamente significativo un valor de $p<0,05$. Para identificar los factores de riesgo se utilizó el Riesgo Relativo (RR) con un intervalo de confianza al 95\%. Finalmente, se estimó el hazard ratio (HR) de las variables que ajustaron en el análisis bivariado a partir de la regresión de Cox y se utilizó el método de Efron para manejar los fracasos concomitantes. Para comparar la ocurrencia de un evento (tiempo de negativización) se empleó el método de LogRank test. Esta base fue analizada en el programa Epi Info 7.0.9.34.

\section{CONSIDERACIONES ÉTICAS}

El proyecto fue aprobado por el Comité de Ética de la Facultad de Ciencias de la Salud de la Universidad Peruana de Ciencias Aplicadas (UPC) con el N PI02812 y por la Oficina de Capacitación, Investigación y Docencia de la Red Asistencial de Rebagliati de Lima. Dado que el estudio se basó en fuente secundaria y no hubo contacto con los pacientes no se aplicó un consentimiento informado; sin embargo, se protegió en todo momento el nombre del paciente.

\section{RESULTADOS}

En el estudio se incluyeron a 31 expuestos y 144 no expuestos. El promedio de edad entre los expuestos fue $51,8 \pm 13,9$ años, mediana de 51 años (RIC 24-78) y el promedio de edad entre los no expuestos fue 45,6, \pm 18,8 años, mediana de 45 años (RIC 12-90).

Las diferencias en las características diagnósticas y clínicas de los expuestos y no expuestos se muestran
Tabla 1. Características de los pacientes con tuberculosis según presencia de diabetes mellitus, Red Asistencial Rebagliati, Lima 2010-2014

\begin{tabular}{|c|c|c|c|}
\hline Variable & $\frac{\text { Con diabetes }}{n=31(\%)}$ & $\frac{\text { Sin diabetes }}{n=144(\%)}$ & $\begin{array}{c}\text { Valor } \\
p^{*}\end{array}$ \\
\hline \multicolumn{4}{|l|}{$\overline{\text { Sexo }}$} \\
\hline Varones & $16(51,6)$ & $73(50,7)$ & 0,916 \\
\hline Mujeres & $15(8,4)$ & $71(49,3)$ & \\
\hline \multicolumn{4}{|l|}{ Edad } \\
\hline$<45$ años & $9(29,0)$ & $71(49,3)$ & 0,063 \\
\hline$\geq 45$ años & $22(71,0)$ & $73(50,7)$ & \\
\hline \multicolumn{4}{|l|}{ Distrito de procedencia } \\
\hline Lima centro & $23(74,2)$ & $111(77,1)$ & 0,911 \\
\hline $\begin{array}{l}\text { Otros distritos de } \\
\text { Lima y Callao }\end{array}$ & $8(25,8)$ & $35(22,9)$ & \\
\hline \multicolumn{4}{|l|}{ Diagnostico } \\
\hline BK (+) & $23(74,2)$ & $135(93,7)$ & 0,002 \\
\hline Cultivo (+) & $8(25,8)$ & $9(6,3)$ & \\
\hline $\begin{array}{l}\text { Número de síntomas: } \\
\text { Promedio (DE) }\end{array}$ & $2,8(1,1)$ & $2,3(1,4)$ & $0,023^{\star *}$ \\
\hline \multicolumn{4}{|l|}{ Cavitaciones } \\
\hline Sí & $15(48,4)$ & $7(4,9)$ & $<0,001$ \\
\hline No & $16(51,6)$ & $137(95,1)$ & \\
\hline \multicolumn{4}{|l|}{ Patrón de resistencia } \\
\hline $\begin{array}{l}\text { Tuberculosis } \\
\text { multidrogorresistente }\end{array}$ & $4(12,9)$ & $3(2,1)$ & 0,022 \\
\hline $\begin{array}{l}\text { Tuberculosis } \\
\text { pulmonar sensib }\end{array}$ & $27(87,1)$ & $141(97$ & \\
\hline
\end{tabular}

DE: Desviación estándar

* Las comparaciones fueron hechas usando la prueba de Chi cuadrado y Test exacto de Fisher.

** Prueba de U de Mann-Whitney

en la Tabla 1. Los síntomas que se evaluaron fueron: tos, sudoración nocturna, expectoración, pérdida de peso, malestar general, pérdida de apetito, dolor torácico, hemoptisis y otros. Se encontraron diferencias estadísticas significativas $(p<0,05)$ entre los pacientes con TB+DM2 y los pacientes con TB en el método de diagnóstico, promedio de síntomas y en el patrón de resistencia de TB. Asimismo, respecto a la cavitación existen diferencias significativas entre los pacientes con TB+DM2 y con TB pulmonar.

Tabla 2. Resultados del tratamiento antituberculoso en pacientes con y sin diabetes mellitus tipo 2 atendidos en la Red Asistencial Rebagliati. Lima 2010-2014

\begin{tabular}{|c|c|c|c|c|}
\hline \multirow{2}{*}{ Variables } & Con diabetes & Sin diabetes & \multirow{2}{*}{ RR (IC 95\%) } & \multirow{2}{*}{ Valor $p^{*}$} \\
\hline & $n=34(\%)$ & $n=133(\%)$ & & \\
\hline \multicolumn{5}{|l|}{ Reacciones adversas al tratamiento antituberculoso } \\
\hline Sí & $4(12,9)$ & $8(5,6)$ & $1,2(0,8-1,8)$ & 0,291 \\
\hline No & $27(87,1)$ & $136(94,4)$ & & \\
\hline \multicolumn{5}{|l|}{ Cambio de esquema de tratamiento } \\
\hline Sí & $13(41,9)$ & $1(0,7)$ & $12,4(1,8-82,2)$ & $<0,001$ \\
\hline No & $18(58,1)$ & $143(99,3)$ & & \\
\hline Duración del tratamiento en meses: promedio (DE) & $6(0)$ & $6,4(1,1)$ & & $<0,001^{* *}$ \\
\hline \multicolumn{5}{|l|}{ Resultado de tratamiento } \\
\hline Fracaso & $11(35,5)$ & $3(2,1)$ & $4,1(1,4-11,1)$ & $<0,001$ \\
\hline Curado & $20(64,5)$ & $141(97,9)$ & & \\
\hline \multicolumn{5}{|l|}{ Negativización del esputo } \\
\hline$\leq 4$ meses & $14(45,2)$ & $2(1,4)$ & $7,1(1,9-26,1)$ & $<0,001$ \\
\hline$>3$ meses & $17(54,8)$ & $142(98,6)$ & & \\
\hline
\end{tabular}

DE: Desviación estándar.

* Prueba exacta de Fisher.

** Prueba de U de Mann-Whitney. 
Tabla 3. Asociación entre diabetes y negativización de esputo en pacientes con tuberculosis pulmonar. Modelos de Regresión de Cox crudo y ajustado

\begin{tabular}{lccccc}
\hline \multirow{2}{*}{ Diabetes } & HR crudo & & HR ajustado* & & HR ajustado** \\
\cline { 2 - 2 } \cline { 5 - 5 } & (IC 95\%) & & (IC 95\%) & (IC 95\%) \\
\hline No & Referencia & & Referencia & Referencia \\
Sí & $4,00(6,25-2,5)$ & & $4,76(7,69-2,9)$ & $4,16(1,1-1,6)$ \\
\hline
\end{tabular}

Se utilizó el método de Efron para manejar los fracasos concomitantes * Modelo ajustado por sexo, edad y distrito de procedencia

** Modelo ajustado por sexo, edad, distrito de procedencia, presencia

de cavitación, patrón de resistencia de TB y cambio de tratamiento

* Log-rank $(p<0,001)$

Respecto a los resultados del tratamiento antituberculoso se encontraron diferencias entre los pacientes con diabetes y sin diabetes para el cambio en el esquema, duración del tratamiento, resultado del tratamiento y negativización del esputo (Tabla 2). En el modelo de regresión de Cox ajustado por sexo, edad, distrito de procedencia, presencia de cavitación, patrón de resistencia de TB y cambio de tratamiento, se identificó que el grupo de pacientes con TB+DM2 retrasaron la negativización del BK de esputo (RR 4,16; IC 95\%: 1,11,6) (Tabla 3), esto significa que el riesgo para persistir como positivo en los pacientes con TB+DM2 es cuatro veces comparado al que tiene únicamente TB.

Los pacientes con TB+DM2 tienen un mayor tiempo de negativización del BK de esputo en comparación con los pacientes sin DM2, los pacientes con DM2 negativizan a partir del cuarto mes, mientras que los pacientes sin DM2 desde el segundo mes (Figura 1).

\section{DISCUSIÓN}

En el presente estudio los pacientes expuestos (TB+DM2) negativizan en un tiempo más largo comparado con los pacientes TB pulmonar, con mayor riesgo de cambiar el esquema del tratamiento, requieren mayor tiempo de tratamiento y el riesgo de fracaso se incrementa

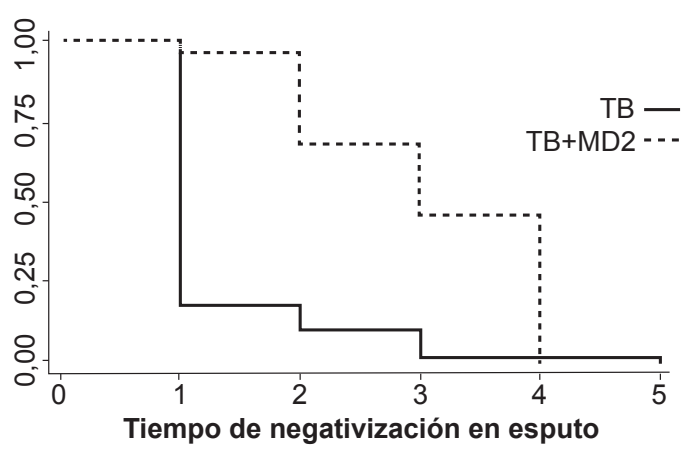

Figura 1. Curva Kaplan-Meier mostrando tiempo en meses hasta negativización de esputo en pacientes con tuberculosis (TB) y tuberculosis con diabetes mellitus tipo 2 (TB+MD2) notablemente. Estos hallazgos también han sido identificados en otros estudios ${ }^{(9,20-21)}$. Uno de los efectos más importantes del alargamiento de la negativización es que incrementan el riesgo de transmisión y elevan el costo del tratamiento al requerir mayor tiempo con medicamentos. Además, el hecho de tener TB+DM2 retrasa la negativización del BK de esputo, corroborado por el análisis de regresión y después de controlar algunos confusores como la edad, sexo, distrito de procedencia, patrón de resistencia de TB y cambio de tratamiento. Resultados similares han sido publicados en cohorte de pacientes de Indonesia ${ }^{(20)}$.

En nuestro estudio encontramos que los pacientes con TB+DM2 presentaron menos síntomas que los pacientes solo con tuberculosis pulmonar, lo que se contrapone con cierta literatura donde no encuentran diferencias significativas en la presentación de los síntomas en los pacientes diabéticos ${ }^{(26-28)}$. Por otro lado, un estudio realizado en la frontera entre México y los Estados Unidos de América mostró que los pacientes diabéticos presentaron mayor frecuencia de fiebre y hemoptisis (29). En Indonesia un estudio reveló que los pacientes diabéticos presentaban más síntomas y signos comparado con los no diabéticos, pero que ésta no implicaba mayor gravedad de la tuberculosis ${ }^{(20)}$.

La edad, es considerado como factor de riesgo para el desarrollo del binomio TB+DM2 (30,31). Sin embargo, existen estudios donde se señala que existe mayor asociación del desarrollo TB+DM2 en pacientes más jóvenes por lo que sigue siendo controvertido si la edad es un factor de riesgo para esta condición ${ }^{(13,32)}$ o es una variable confusora.

No existe un patrón patognomónico que la señale; sin embargo, un estudio en la India señala que pacientes con diabetes presentan una mayor incidencia de cavitaciones a nivel radiológico ${ }^{(33)}$. El período de negatividad del frotis bronquial fue mayor entre las personas con TB+DM2, al compararlos con los que no presentan diabetes, el mismo resultado fue hallado en otros estudios los cuales toman esta medida como una variable importante debido a sus repercusiones al momento de una decisión terapéutica al finalizar la fase 1 del tratamiento esquema 1 en pacientes tratados por TB pulmonar sensible. No se encontró estudios que relacionen esta variable en pacientes diabéticos.

En cuanto al resultado del tratamiento la mayoría de pacientes catalogados como fracaso seencontraron dentro del grupo de pacientes con TB+DM2. Investigaciones previas señala que este grupo de pacientes tiene un riesgo más elevado de desarrollar esta condición debido a la inmunosupresión presente en la enfermedad (15). Asimismo, no se encontró evidencia significativa para 
la RAFA y su asociación en el grupo de pacientes con TB+DM2. Sin embargo, cabe resaltar que esta reacción fue menor con el grupo expuesto, es probable que pueda haber algún sesgo en el registro de las RAFA por tratarse de un estudio retrospectivo. Cabe resaltar que el número de sujetos con TB+DM2 es reducido.

El cambio de tratamiento fue mayor en pacientes del grupo TB+DM2. Esta variable no ha sido considerada en otras investigaciones; sin embargo, es un factor importante en el manejo de este tipo de pacientes puesto que la diabetes puede modificar la negativización del BK, lo cual no necesariamente refleja una falla en el tratamiento, asimismo, la falta de un esquema para este tipo de pacientes hace que los médicos tratantes tomen conductas distintas en el manejo farmacológico.

La diabetes es una de las comorbilidades más frecuentemente asociadas a la infección por TB pulmonar ${ }^{(34)}$. Nuestro estudio encuentra diferencias en cuanto a las manifestaciones clínicas, radiológicas y bacteriológicas que alteran el curso de la enfermedad de ahí la importancia de un esquema de tratamiento específico para esta condición. En Brasil la Sociedad Brasilera de Neumología recomienda en su última guía de manejo de tuberculosis considerar un esquema de 9 meses a los pacientes que presenten la asociación TB+DM2 ${ }^{(35)}$.

La selección de los no expuestos ha sido por conveniencia, lo que impide la extrapolación de los resultados. Dado que el estudio se basó en fuente secundaria, los datos descritos en la historia clínica, dependen del criterio del radiólogo y clínico asumiendo que sea correcta la descripción de la radiografía de tórax y la realización de una correcta anamnesis de los pacientes estudiados, este hecho puede haber tenido algún efecto en los resultados. La utilización del BK de esputo como criterio diagnóstico, debido a su baja sensibilidad en la detección de tuberculosis en pacientes con baja carga bacilar ${ }^{(36)}$. No se logró encontrar los motivos del cambio del esquema del tratamiento ni los datos completos de la variación del peso corporal. Los valores de glicemia durante el tratamiento antituberculoso no pudieron ser recolectados pues no están considerados en la hoja de control del PCT. Finalmente, no se registró la conducta tomada para los pacientes catalogados como fracaso, pues no está contemplada como objetivo de este estudio.

Es importante que se realicen otros estudios para conocer mejor el binomio TB+DM2 con un diseño de cohorte prospectiva considerando las modificaciones que han sido incorporadas en la norma nacional de tuberculosis ${ }^{(24)}$.

En el presente estudio los pacientes TB+DM2 tardan dos meses más en negativizarla baciloscopía comparado con los pacientes TB pulmonar, asimismo, la presencia de cavitación es mayor en los pacientes con TB+DM2.

Existen diferencias significativas en el cambio en el esquema del tratamiento, resultados y duración del tratamiento entre los expuestos (TB+DM2) y los no expuestos (TB pulmonar). Asimismo, existen diferencias estadísticas entre los pacientes con TB+DM2 y los pacientes con TB en cuanto a la edad, la mediana en el número de síntomas y en la forma clínica de la TB.

\begin{abstract}
Contribuciones de autoría: Todos los autores han participado en la concepción, diseño y aprobación de versión final del artículo. OCT, PCS y FEDA participaron en la recolección de datos/obtención de resultados, redacción del artículo. NYC realizó el análisis e interpretación de datos, además contribuyó a la redacción. JWTS realizó la revisión crítica del artículo.
\end{abstract}

Fuentes de financiamiento. Autofinanciado.

Conflictos de interés: Los autores declaramos no tener conflicto de interés en la publicación del artículo.

\section{REFERENCIAS BIBLIOGRÁFICAS}

1. Zumla A, Raviglione M, Hafner R, von Reyn F. Tuberculosis. N Engl J Med. 2013;368(8):745-55. doi: 10.1056/ NEJMra1200894.

2. Caminero JA, editor. Guidelines for clinical and operational management of drug-resistant tuberculosis. Paris: International Union Against Tuberculosis and Lung Disease; 2013.

3. World Health Organization (WHO). Global Tuberculosis Report 2013. Ginebra: WHO; 2013.

4. Ministerio de Salud de Perú. Estrategia Sanitaria Nacional de Prevención y Control de la Tuberculosis. Sala situacional de TB Lima: MINSA; 2012 [citado el 7 de diciembre de 2014]. Disponible en: http://190.223.45.115/ newtb/Archivos/RecursoInformacion/20140211115135.pdf

5. Lönnroth $\mathrm{K}$, Castro KG, Chakaya JM, Chauhan LS, Floyd K, Glaziou $\mathrm{P}$, et al. Tuberculosis control and elimination 2010-50: cure, care, and social development. Lancet. $2010 ; 375(9728): 1814-29$. doi: 10.1016/S0140-6736(10)60483-7.

6. Jeon CY, Murray MB. Diabetes mellitus increases the risk of active tuberculosis: a systematic review of 13 observational studies. PLoS Med. 2008;5(7):e152. doi: 10.1371/journal.pmed.0050152.
7. Magee MJ, Foote M, Maggio DM, Howards PP, Narayan KM, Blumberg HM, et al. Diabetes mellitus and risk of all-cause mortality among patients with tuberculosis in the state of Georgia, 2009-2012. Ann Epidemiol. 2014;24(5):369-75. doi: 10.1016/j.annepidem.2014.01.012.

8. Uchimura K, Ngamvithayapong-Yanai J, Kawatsu L, Ohkado A, Yoshiyama T, Shimouchi A, et al. Characteristics and treatment outcomes of tuberculosis cases by risk groups, Japan, 2007 2010. Western Pac Surveill Response J. 2013;4(1):11-8. doi: 10.5365/WPSAR.2012.3.4.016. 
9. Jiménez-Corona ME, Cruz-Hervert LP, García-García L, Ferreyra-Reyes L, Delgado-Sánchez G, Bobadilla-Del-Valle $\mathrm{M}$, et al. Association of diabetes and tuberculosis: impact on treatment and post-treatment outcomes. Thorax. 2013;68(3):214-20. doi: 10.1136/thoraxjnl-2012-201756.

10. Magee MJ, Bloss E, Shin SS, Contreras C, Huaman HA, Ticona JC, et al. Clinical characteristics, drug resistance, and treatment outcomes among tuberculosis patients with diabetes in Peru. Int J Infect Dis. 2013;17(6):e404-12. doi: 10.1016/j.ijid.2012.12.029.

11. Guariguata L, Whiting DR, Hambleton I, Beagley J, Linnenkamp U, Shaw JE. Global estimates of diabetes prevalence for 2013 and projections for 2035. Diabetes Res Clin Pract. 2014;103(2):137-49. doi: 10.1016/j. diabres.2013.11.002.

12. Aschner P, Aguilar-Salinas C, Aguirre L, Franco L, Gagliardino JJ, de Lapertosa SG, et al. Diabetes in South and Central America: an update. Diabetes Res Clin Pract. 2014;103(2):238-43. doi: 10.1016/j.diabres.2013.11.010.

13. Ponce-De-Leon A, Garcia-Garcia Md Mde L, Garcia-Sancho MC, Gomez-Perez FJ, Valdespino-Gomez JL, Olaiz-Fernandez G, et al. Tuberculosis and diabetes in southern Mexico. Diabetes Care. 2004;27(7):1584-90.

14. Carreira S, Costeira J, Gomes C, André JM, Diogo N. Impacto da diabetes na forma de apresentação da tuberculoseemdoentes hospitalizados. Rev Port Pneumol. 2012;18(5):239-43. doi: 10.1016/j.rppneu.2012.04.001.

15. Perez-Guzman C, Torres-Cruz A, Villareal-Velarde H, Vargas MH. Progressive age-related changes in pulmonary tuberculosis images and the effect of diabetes. Am J Respir Crit Care Med. 2000;162(5):1738-40.

16. Oursler KK, Moore RD, Bishai WR, Harrington SM, Pope DS, Chaisson RE. Survival of patients with pulmonary tuberculosis: clinical and molecular epidemiologic factors. Clin Infect Dis. 2002;34(6):752-9.

17. Tatar D, Senol G, Alptekin S, Karakurum C, Aydin M, Coskunol I. Tuberculosis in diabetics: features in an endemic area. Jpn J Infect Dis. 2009;62(6):423-7.

18. Dobler CC, Flack JR, Marks GB. Risk of tuberculosis among people with diabetes mellitus: an Australian nationwide cohort study. BMJ Open. 2012;2(1):e000666. doi: 10.1136/bmjopen-2011-000666.

19. Patel AK, Rami KC, Ghanchi FD. Radiological presentation of patients of pulmonary tuberculosis with diabetes mellitus. Lung India. 2011;28(1):70. doi: $10.4103 / 0970-2113.76308$.

20. Alisjahbana B, Sahiratmadja E, Nelwan EJ, Purwa AM, Ahmad Y, Ottenhoff $\mathrm{TH}$, et al. The effect of type 2 diabetes mellitus on the presentation and treatment response of pulmonary tuberculosis. Clin Infect Dis. 2007;45(4):42835.

21. Dooley KE, Chaisson RE. Tuberculosis and diabetes mellitus: convergence of two epidemics. Lancet Infect Dis. 2009;9(12):737-46. doi: 10.1016/ S1473-3099(09)70282-8.

22. Delgado Rospigliosi JL, Seclen Santisteban SN, Gotuzzo Herencia E. Tuberculosis en pacientes con diabetes mellitus: Un estudio epidemiológico y clínico en el Hospital Nacional Cayetano Heredia. Rev Med Hered. 2006;17(3):132-40.

23. Ugarte-Gil C, Moore DAJ. Comorbilidad de tuberculosis y diabetes: problema aún sin resolver. Rev Peru Med Exp Salud Publica. 2014;31(1):137-42.

24. Ministerio de Salud de Perú. Norma técnica de salud para la atención integral de las personas afectadas por tuberculosis. NT No 104-MINSA/ DGSP.v.01. RM No 715-2013/MINSA. Lima: MINSA; 2013.

25. American Diabetes Association. Diagnosis and classification of diabetes mellitus. Diabetes Care. 2013;36(Suppl 1):S67-74. doi: $10.2337 / \mathrm{dc13}-$ S067.

26. Nissapatorn V, Kuppusamy I, Jamaiah I, Fong MY, Rohela M, Anuar AK. Tuberculosis in diabetic patients: a clinical perspective. Southeast Asian J Trop Med Public Health. 2005;36(Suppl 4):213-20.

27. Singla R, Khan N, Al-Sharif N, Ai-Sayegh MO, Shaikh MA, Osman MM. Influence of diabetes on manifestations treatment outcome of pulmonary TB patients. Int J Tuberc Lung Dis. 2006;10(1):74-9.

28. Bacakoğlu F, Başoğlu OK, Cok G, Sayiner A, Ateş M. Pulmonary tuberculosis in patients with diabetes mellitus. Respiration. 2001;68(6):595-600.
29. Restrepo BI, Fisher-Hoch SP, Crespo JG, Whitney E, Perez A, Smith B, et al. Type 2 diabetes tuberculosis in a dynamic bi-national border population. Epidemiol Infect 2007;135(3):483-91.

30. Goldhaber-Fiebert JD, Jeon CY, Cohen T, Murray MB. Diabetes mellitus and tuberculosis in countries with high tuberculosis burdens: individual risks and social determinants. Int J Epidemiol. 2011; 40(2):417-28. doi: $10.1093 /$ ije/dyq 238.

31. Pérez-Navarro LM, Fuentes-Domínguez F, Morales-Romero J, Zenteno-Cuevas R. Factores asociados a tuberculosis pulmonar en pacientes con diabetes mellitus de Veracruz, México. Gaceta Méd México. 2011;147:21925.

32. Kim SJ, Hong YP, Lew WJ, Yang SC, Lee EG. Incidence of pulmonary tuberculosis among diabetics. Tuber Lung Dis. 1995;76(6):529-33.

33. Alavi SM, Khoshkho MM, Salmanzadeh S, Eghtesad M. Comparison of epidemiological, clinical, laboratory and radiological features of hospitalized diabetic and non-diabetic patients with pulmonary tuberculosis at razi hospital in ahvaz. Jundishapur J Microbiol. 2014;7(9):e12447. doi: 10.5812/ jjm.12447.

34. Baghaei P, Marjani M, Javanmard P, Tabarsi P, Masjedi MR. Diabetes mellitus and tuberculosis facts and controversies. J Diabetes Metab Disord. 2013;12(1):58. doi: 10.1186/22516581-12-58.

35. Conde MB, Melo FA, Marques AM, Cardoso NC, Pinheiro VG, Dalcin Pde T, et al. III Diretrizes para Tuberculose da Sociedade Brasileira de Pneumologia e Tisiologia. J Bras Pneumol. 2009;35(10):1018-48.

36. Parsons LM, Somoskövi A, Gutierrez C, Lee E, Paramasivan CN, Abimiku $\mathrm{A}$, et al. Laboratory diagnosis of tuberculosis in resource-poor countries: challenges and opportunities. Clin $\mathrm{Mi}$ crobiol Rev. 2011;24(2):314-50. doi: 10.1128/CMR.00059-10.

Correspondencia: Frank Enrique De La Cruz Armijo

Dirección: Av. Guillermo de la Fuente n650

- Comas, Lima, Perú.

Teléfono: 51941465397

Correo electrónico:fda1415@gmail.com 